\title{
Characterization of the Hysteresis Duhem Model ${ }^{\star}$
}

\author{
Mohammad Fuad Mohammad Naser*, Fayçal Ikhouane** \\ Universitat Politècnica de Catalunya, Escola Universitària \\ d'Enginyeria, Tècnica Industrial de Barcelona. Departament de \\ Matemàtica Aplicada III, Comte d’Urgell, 187, 08036, Barcelona, \\ Spain \\ *mohammad.fuad@upc.edu \\ **faycal.ikhouane@upc.edu
}

\begin{abstract}
The Duhem model, widely used in structural, electrical and mechanical engineering, gives an analytical description of a smooth hysteretic behavior. In practice, the Duhem model is mostly used within the following black-box approach: given a set of experimental input-output data, how to tune the model so that its output matches the experimental data. It may happen that a Duhem model presents a good match with the experimental real data for a specific input, but does not necessarily keep significant physical properties which are inherent to the real data, independently of the exciting input. This paper presents a characterization of different classes of Duhem models in terms of their consistency with the hysteresis behavior.
\end{abstract}

Keywords: mathematical model; hysteresis; convergence; stability; operator

\section{INTRODUCTION}

Hysteresis is a nonlinear behavior encountered in a wide variety of processes including biology, optics, electronics, ferroelectricity, magnetism, mechanics, structures, among other areas. The detailed modeling of hysteresis systems using the laws of Physics is an arduous task, and the obtained models are often too complex to be used in applications. For this reason, alternative models of these complex systems have been proposed $[24 ; 5 ; 15 ; 12 ; 18]$. These models do not come, in general, from the detailed analysis of the physical behavior of the systems with hysteresis. Instead, they combine some physical understanding of the system along with some kind of black-box modeling.

One of the popular models for hysteresis is the Duhem model proposed in [7]. The generalized form of the Duhem model consists of an ordinary differential equation of the form $\dot{x}=f(x, u) g(\dot{u})$, where $u$ is the input and $x$ is the state or the output [22]. Other special forms of the model have been used, like the form $\dot{x}=f_{1}(x, u) \max \{\dot{u}, 0\}+f_{2}(x, u) \min \{\dot{u}, 0\}$ [17] or the semilinear form $\dot{x}=(A x+B u) g(\dot{u})$ [19]. Other important special cases of the Duhem model are the LuGre model of friction [26], the Dahl model of friction [6] and the BoucWen model of hysteresis $[25 ; 4]$. The Duhem model has been used to represent friction [22], electromagnetic behavior $[9 ; 10]$, or hysteresis in magnetorheological dampers [23].

\footnotetext{
* Supported by grant DPI2011-25822 of the Spanish Ministry of Economy and Competitiveness.
}

In the current literature, the Duhem model is mostly used within the following black-box approach: given a set of experimental input-output data, how to adjust the Duhem model so that the output of the model matches the experimental data? The use of system identification techniques is one practical way to perform this task. Once an identification method has been applied to tune the Duhem model, the resulting model is considered as a "good" approximation of the true hysteresis when the error between the experimental data and the output of the model is small enough. Then this model is used to study the behavior of the true hysteresis under different excitations. By doing this, it is important to consider the following remark. It may happen that a Duhem model presents a good match with the experimental real data for a specific input, but does not necessarily keep significant physical properties which are inherent to the real data, independently of the exciting input. In the current literature, this issue has been considered in $[13 ; 20]$ regarding the passivity/dissipativity of Duhem model.

In this paper, we investigate the conditions under which the Duhem model is consistent with the hysteresis behavior. The concept of consistency is formalized in [11] where a general class of hysteresis operators is considered. From the results of [11], it can be concluded that, to check consistency one has to consider the sequence of inputs $u_{\gamma}(t)=u(t / \gamma), t \geq 0, \gamma>0$ and the corresponding sequence of outputs $x_{\gamma}$ with $\dot{x}_{\gamma}=f\left(x_{\gamma}, u_{\gamma}\right) g\left(\dot{u}_{\gamma}\right)$. For the Duhem model to represent a hysteresis system, it is necessary that the sequence of functions $t \rightarrow x_{\gamma}(\gamma t)$ converge uniformly when $\gamma \rightarrow \infty$. In this paper, we seek necessary conditions and sufficient ones for this uniform convergence to hold. 


\section{PROBLEM STATEMENT}

The generalized Duhem model is defined for almost all $t \geq 0$ by $[22]$

$$
\begin{aligned}
& \dot{x}(t)=f(x(t), u(t)) g(\dot{u}(t)), \\
& x(0)=x_{0},
\end{aligned}
$$

where $x_{0}$ and state $x(t)$ take values in $\mathbb{R}^{m}$ for some positive integer $m$, input $u \in W^{1, \infty}\left(\mathbb{R}_{+}, \mathbb{R}\right)^{1}$, function $f: \mathbb{R}^{m} \times$ $\mathbb{R} \rightarrow \mathbb{R}^{m \times r}$ is continuous, where $r$ and $m$ are positive integers. Finally, $g: \mathbb{R} \rightarrow \mathbb{R}^{r}$ is continuous and satisfies $g(0)=\mathbf{0}$.

Since $g$ is continuous and $\dot{u} \in L^{\infty}\left(\mathbb{R}_{+}, \mathbb{R}\right)$, we have $g \circ \dot{u} \in L^{\infty}\left(\mathbb{R}, \mathbb{R}^{r}\right)$. The differential equation (1) satisfies Carathéodory conditions, thus, for each initial state $x_{0} \in \mathbb{R}^{m},(1)$ has an absolutely continuous solution that is defined on an interval of the form $[0, T), T>0[8, \mathrm{p} .4]$. In this paper, we assume that the system (1)-(2) has a unique solution.

Consider the time scale change $s_{\gamma}(t)=t / \gamma, \gamma>0, t \geq 0$. When the input $u \circ s_{\gamma}$ is used instead of $u$, the system (1)-(2) becomes

$$
\begin{aligned}
& \dot{x}_{\gamma}(t)=f\left(x_{\gamma}(t), u \circ s_{\gamma}(t)\right) g\left(\frac{1}{\gamma} \dot{u} \circ s_{\gamma}(t)\right) \\
& x_{\gamma}(0)=x_{0} .
\end{aligned}
$$

When $\gamma=1$, system (3)-(4) reduces to (1)-(2). For any $\gamma>0$, define $\sigma_{\gamma}: \mathbb{R}_{+} \rightarrow \mathbb{R}^{m}$ as $\sigma_{\gamma}=x_{\gamma} \circ s_{1 / \gamma}$. System (3)-(4) can be re-written as:

$$
\sigma_{\gamma}(t)=x_{0}+\gamma \int_{0}^{t} f\left(\sigma_{\gamma}(\tau), u(\tau)\right) g\left(\frac{1}{\gamma} \dot{u}(\tau)\right) d \tau
$$

for all $\gamma>0$ and for almost all $t \in\left[0, \omega_{\gamma}\right)$, where $\left[0, \omega_{\gamma}\right)$ is the maximal interval of existence of the solution $\sigma_{\gamma}$.

Definition 2.1. In the system (1)-(2), the operator which maps $\left(u, x_{0}\right)$ to $x$ is said to consistent if the function $\sigma_{\gamma}$ converges in $L^{\infty}[11]$.

It is shown in [11], that for hysteresis process, the sequence of functions $\left\{\varphi_{u \circ s_{\gamma}}\right\}_{\gamma>0}$ converges in $L^{\infty}\left(I_{u}, \mathbb{R}^{m}\right)$ as $\gamma \rightarrow$ $\infty$. This fact shows that consistency is a mathematical property that any model of hysteresis should satisfy.

\section{NECESSARY CONDITIONS}

Definition 3.1. Let $G \in C^{0}\left(\left[t_{1}, t_{2}\right], \mathbb{R}\right)$. We define the right and left local fractional derivative of $G$ at $t_{3} \in\left(t_{1}, t_{2}\right)$ with respect to order $\lambda>0$ respectively as [2]:

$$
D_{+}^{\lambda} G\left(t_{3}\right)=\Gamma(1+\lambda) \lim _{\kappa \rightarrow t_{3}+} \frac{G(\kappa)-G\left(t_{3}\right)}{\left(\kappa-t_{3}\right)^{\lambda}},
$$

$1 W^{1, \infty}\left(\mathbb{R}_{+}, \mathbb{R}\right)$ is the Sobolev space of absolutely continuous functions $u: \mathbb{R}_{+} \rightarrow \mathbb{R}$. For this class of functions, the derivative $\dot{u}$ is defined a.e. with $\|u\|_{\infty}<\infty$ and $\|\dot{u}\|_{\infty}<\infty$. Endowed with the norm $\|u\|_{1, \infty}=\max \left(\|u\|_{\infty},\|\dot{u}\|_{\infty}\right), W^{1, \infty}\left(\mathbb{R}_{+}, \mathbb{R}\right)$ is a Banach space [1]

$$
D_{-}^{\lambda} G\left(t_{3}\right)=\Gamma(1+\lambda) \lim _{\kappa \rightarrow t_{3}-} \frac{G\left(t_{3}\right)-G(\kappa)}{\left(t_{3}-\kappa\right)^{\lambda}},
$$

where $\Gamma$ is the gamma function.

The local fractional derivatives of vector-valued functions can be differentiated by simply differentiating the components in the Cartesian coordinate system.

Definition 3.2. The function $g \in C^{0}\left(\mathbb{R}, \mathbb{R}^{r}\right)$ is said to be of class $\lambda>0$ if the quantities $D_{+}^{\lambda} g(0), D_{-}^{\lambda} g(0)$ exist, are finite, and at least one of them is nonzero.

Proposition 3.1. The function $g \in C^{0}\left(\mathbb{R}, \mathbb{R}^{r}\right)$ is of class $\lambda$ if and only if

$$
\forall \vartheta \in \mathbb{R}, \text { we have } \lim _{\gamma \rightarrow \infty} \gamma^{\lambda} g\left(\frac{\vartheta}{\gamma}\right)=g^{*}(\vartheta),
$$

where $g^{*} \in C^{0}\left(\mathbb{R}, \mathbb{R}^{r}\right)$ is defined as

$$
g^{*}(\vartheta)= \begin{cases}\vartheta^{\lambda} \lim _{\kappa \rightarrow 0+} \frac{g(\kappa)}{\kappa^{\lambda}} & \vartheta \geq 0 \\ (-\vartheta)^{\lambda} \lim _{\kappa \rightarrow 0-} \frac{g(\kappa)}{(-\kappa)^{\lambda}} & \vartheta<0\end{cases}
$$

Lemma 3.1. Assume that the system (1)-(2) has a unique global solution ${ }^{2}$ for each input $u \in W^{1, \infty}\left(\mathbb{R}_{+}, \mathbb{R}\right)$ and initial condition $x_{0} \in \mathbb{R}^{m}$. Assume that the function $g$ is of class $\lambda>0$. Suppose that there exists a continuous function $Q: \mathbb{R}_{+} \times \mathbb{R}_{+} \times \mathbb{R}_{+} \rightarrow \mathbb{R}_{+}$such that

$$
|x(t)| \leq Q\left(\left|x_{0}\right|,\|u\|_{\infty},\|\dot{u}\|_{\infty}\right), \forall t \geq 0
$$

for each initial state $x_{0} \in \mathbb{R}^{m}$ and each input $u \in$ $W^{1, \infty}\left(\mathbb{R}_{+}, \mathbb{R}\right)$. Assume that the system (1)-(2) is consistent with respect to $\left(u, x_{0}\right)$; that is there exists $q_{u} \in$ $L^{\infty}\left(\mathbb{R}_{+}, \mathbb{R}^{m}\right)$ such that $\lim _{\gamma \rightarrow \infty}\left\|\sigma_{\gamma}-q_{u}\right\|_{\infty}=0$, then

if $\lambda=1$, we have

- $q_{u} \in W^{1, \infty}\left(\mathbb{R}_{+}, \mathbb{R}^{m}\right)$.

- We have for all $t \geq 0$ that

$$
q_{u}(t)=x_{0}+\int_{0}^{t} f\left(q_{u}(\tau), u(\tau)\right) g^{*}(\dot{u}(\tau)) d \tau,
$$

where $g^{*}$ is given in equation (6).

if $\lambda \in(0,1)$, we have

- $q_{u} \in C^{0}\left(\mathbb{R}_{+}, \mathbb{R}^{m}\right) \cap L^{\infty}\left(\mathbb{R}_{+}, \mathbb{R}^{m}\right)$.

- $q_{u}(0)=x_{0}$.

\footnotetext{
2 The standard way to ensure that the system (1)-(2) admits a unique solution is to prove that the right-hand side of (1)-(2) is Lipschitz with respect to $x$. A function $\nu: D \subseteq \mathbb{R}^{m} \times \mathbb{R}_{+} \rightarrow \mathbb{R}^{m}$ is Lipschitz with respect to $x$ if there exists a summable function $l: \mathbb{R}_{+} \rightarrow \mathbb{R}_{+}$such that $\left|\nu\left(\alpha_{1}, t\right)-\nu\left(\alpha_{2}, t\right)\right| \leq l(t)\left|\alpha_{1}-\alpha_{2}\right|$, for almost all $t \geq 0$ and for all $\alpha_{1}, \alpha_{2} \in \mathbb{R}^{m}$ that satisfy $\left(t, \alpha_{1}\right),\left(t, \alpha_{2}\right) \in$ $D[8]$
} 
- We have for almost all $t \geq 0$ that

$$
f\left(q_{u}(t), u(t)\right) g^{*}(\dot{u}(t))=\mathbf{0},
$$

where $g^{*}$ is defined in equation (6).

if $\lambda>1$, we have $q_{u}(t)=x_{0}, \forall t \geq 0$.

\section{SUFFICIENT CONDITIONS}

This section presents sufficient conditions for the consistency for the system (1)-(2) with respect to $\left(u, x_{0}\right)$.

Definition 4.1. [14] A continuous function $\beta: \mathbb{R}_{+} \rightarrow \mathbb{R}_{+}$ is said to belong to class $\mathcal{K}_{\infty}$ if it is increasing, satisfies $\beta(0)=0$, and $\lim _{t \rightarrow \infty} \beta(t)=\infty$.

Lemma 4.1. Suppose that the system (1)-(2) has a unique solution and that the function $g$ is of class $\lambda \in(0,1)$. Assume that there exists $q_{u} \in W^{1, \infty}\left(\mathbb{R}_{+}, \mathbb{R}^{m}\right)$ such that for almost all $t \geq 0$

$$
\left\{\begin{array}{l}
q_{u}(0)=x_{0}, \\
f\left(q_{u}(t), u(t)\right) g^{*}(\dot{u}(t))=\mathbf{0} .
\end{array}\right.
$$

For all $\gamma>0$, define $y_{\gamma}: \mathbb{R}_{+} \rightarrow \mathbb{R}^{m}$ as

$$
y_{\gamma}(t)=\sigma_{\gamma}(t)-q_{u}(t)=x_{\gamma}(\gamma t)-q_{u}(t),
$$

for all $t \in\left[0, \omega_{\gamma}\right)$, where $\left[0, \omega_{\gamma}\right)$ is the maximal interval of existence of solution $\sigma_{\gamma}$ in (5). Suppose that we can find a continuously differentiable function $V: \mathbb{R}^{m} \rightarrow \mathbb{R}_{+}$such that

(1) There exists a function $\delta_{1}: \mathbb{R}_{+} \rightarrow \mathbb{R}_{+}$that satisfies

$$
\delta_{1}(\gamma) \rightarrow 0 \text { as } \gamma \rightarrow \infty \text {. }
$$

(2) There exist constants $\gamma_{*}, \delta_{2}>0$, continuous functions $R_{1}, R_{2}: \mathbb{R}_{+} \rightarrow \mathbb{R}_{+}$and $\mathcal{K}_{\infty}$ class functions $\beta_{1}, \beta_{2}, \beta_{3}:$ $\mathbb{R}_{+} \rightarrow \mathbb{R}_{+}$satisfying:

$$
\beta_{1}(|\alpha|) \leq V(\alpha) \leq \beta_{2}(|\alpha|), \forall \alpha \in \mathbb{R}^{m},
$$

$$
\left\{\begin{array}{l}
\left.\frac{d V(\alpha)}{d \alpha}\right|_{\alpha=y_{\gamma}(t)} \cdot f\left(y_{\gamma}(t)+q_{u}(t), u(t)\right) g\left(\frac{\dot{u}(t)}{\gamma}\right) \\
\leq-\frac{1}{\gamma^{\lambda}} \beta_{3}\left(\left|y_{\gamma}(t)\right|\right)+\frac{1}{\gamma} R_{1}\left(\left|y_{\gamma}(t)\right|\right) \\
\text { for almost all } t \in\left[0, \omega_{\gamma}\right) \text { and } \forall \gamma>\gamma_{*} \\
\text { that satisfy } \delta_{1}(\gamma)<\left|y_{\gamma}(t)\right|<\delta_{2},
\end{array}\right.
$$

$$
\left|\frac{d V(\alpha)}{d \alpha}\right| \leq R_{2}(|\alpha|), \forall \alpha \in \mathbb{R}^{m}
$$

Then,

- There exist $E, \gamma^{*}>0$ such that $\forall \gamma>\gamma^{*}: \omega_{\gamma}=+\infty$, and $\left\|x_{\gamma}\right\|_{\infty} \leq E$, where $x_{\gamma}$ is given in (3)-(4).

- $\lim _{\gamma \rightarrow \infty}\left\|\sigma_{\gamma}-q_{u}\right\|_{\infty}=0$.

Remark 4.1. For $\lambda \in(0,1)$, if the function $q_{u} \in$ $C^{0}\left(\mathbb{R}_{+}, \mathbb{R}^{m}\right) \cap L^{\infty}\left(\mathbb{R}_{+}, \mathbb{R}^{m}\right)$ in Lemma 4.1 is such that $q_{u}=R(u)$ for some $R: \mathbb{R} \rightarrow \mathbb{R}^{m}$, then the graphs $\left\{\left(\sigma_{\gamma}(t), u(t)\right), t \geq 0\right\}$ converge to the curve $R$ as $\gamma \rightarrow \infty$. Hence (1)-(2) is not a hysteresis because the hysteresis loop cannot be a function [3]. This fact is illustrated in the following Example.
Example. Consider the semilinear Duhem model:

$$
\begin{aligned}
\dot{x} & =(A x+B u) g(\dot{u}), \\
x(0) & =x_{0},
\end{aligned}
$$

where $A$ is a Hurwitz $m \times m$ matrix (that is, every eigenvalue of $A$ has negative real part), vector $B$ and state $x$ taking values in $\mathbb{R}^{m}$. The right-hand side of (16) is Lipschitz and thus the system has a unique solution [8]. Take an input $u \in W^{1, \infty}\left(\mathbb{R}_{+}, \mathbb{R}\right)$ such that $A^{-1} B u(0)=-x_{0}$, and that $|\dot{u}(t)| \geq M$ for almost all $t \in \mathbb{R}$ and for some $M>0$. Assume that the function $g: \mathbb{R} \rightarrow \mathbb{R}_{+}$is of class $\lambda \in(0,1)$ and that $d_{+}^{\lambda} g(0), d_{-}^{\lambda} g(0)>0$. The function $q_{u} \in W^{1, \infty}\left(\mathbb{R}_{+}, \mathbb{R}\right)$ which is defined as $q_{u}=-A^{-1} B u$ satisfies (10).

Since $A$ is Hurwitz, there exists a $m \times m$ positive-definite matrix $P$ such that $\left[14\right.$, p.136]: $P A+A^{T} P=-I$, where $I$ is the identity matrix. Consider the continuously differentiable quadratic Lyapunov function candidate $V$ : $\mathbb{R}^{m} \rightarrow \mathbb{R}$ such that $V(\alpha)=\alpha^{T} P \alpha, \forall \alpha \in \mathbb{R}^{m}$. It can be checked that all conditions of Lemma 4.1 are satisfied. Thus, it follows from Lemma 4.1 that there exist some $E, \gamma^{*}>0$ such that $\forall \gamma>\gamma^{*}$, the solution of (16) is global with $\left|x_{\gamma}(t)\right| \leq E, \forall t \geq 0$. Moreover, the operator which maps $\left(u, x_{0}\right)$ to $x$ is consistent. In particular, we have $\left\|\sigma_{\gamma}-q_{u}\right\|_{\infty}=\left\|\sigma_{\gamma}+A^{-1} B u\right\|_{\infty} \rightarrow 0$ as $\gamma \rightarrow \infty$.

As a conclusion, the graphs $\left\{\left(\sigma_{\gamma}(t), u(t)\right), t \geq 0\right\}$ converge to the graph of the linear function $R: \bar{R} \rightarrow \mathbb{R}^{m}$, which is defined as $R(\alpha)=-A^{-1} B \alpha, \forall \alpha \in \mathbb{R}$. This means that for $\lambda \in(0,1)$, the model (16) does not represent a hysteresis (see Remark 4.1).

Simulations: Take $m=1, B=1.0, A=-1.0$, and $x_{0}=0$. Let $g(\vartheta)=\sqrt{|\vartheta|}, \forall \vartheta \in \mathbb{R}$, then $d_{+}^{\lambda} g(0)=d_{-}^{\lambda} g(0)=$ $\Gamma(3 / 2)=\Gamma(1 / 2) / 2=\sqrt{\pi} / 2>0$. Let $u \in W^{1, \infty}\left(\mathbb{R}_{+}, \mathbb{R}\right)$ be the function of period 2 such that $u(t)=t, \forall t \in[0,1]$, and $u(t)=2-t, \forall t \in[1,2]$. Then, we have $|\dot{u}(t)|=$ 1 , for almost all $t \geq 0$. We also have $q_{u}=-A^{-1} B u=u$. Figure 1 left shows that the graph $\left\{\left(\sigma_{\gamma}(t), u(t)\right) / t \geq 0\right\}$ collapses into the identity function when $\gamma \rightarrow \infty$. This happens because of the fact that $q_{u}=u$ and Remark 4.1. Figure 1 right shows that the sequence of functions $\sigma_{\gamma}$ converges uniformly to $q_{u}=u$ as $\gamma \rightarrow \infty$.

\subsection{Class $\lambda=1$ Functions}

In this subsection, we consider class $\lambda=1$ functions.

Proposition 4.1. Assume the following

(1) The system (1)-(2) has a unique global solution.

(2) For the function $g$ in system (1)-(2), there exist $a_{1}, a_{2} \in \mathbb{R}^{r}$ such that

$$
g(\vartheta)= \begin{cases}a_{1} \vartheta & \vartheta \geq 0 \\ a_{2} \vartheta & \vartheta<0\end{cases}
$$

Then the sequence of functions $\sigma_{\gamma}$ of (5) is independent of $\gamma$ and the operator which maps $\left(u, x_{0}\right)$ to $x$ is consistent. 

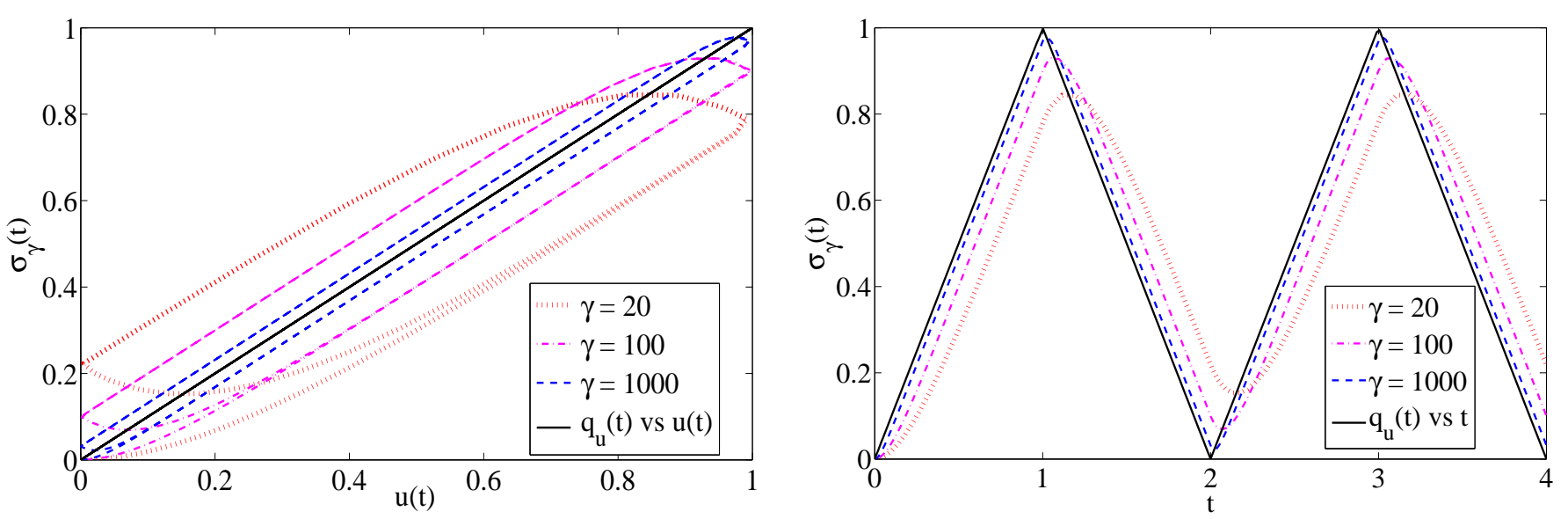

Fig. 1: Left $\sigma_{\gamma}(t)$ versus $u(t)$ for different values of $\gamma$. Right $\sigma_{\gamma}(t)$ versus $t$ for different values of $\gamma$. The plots are for system (16). In each plot, the function $q_{u}$ is the solid line.

Example. Consider Bouc's hysteresis model [4]:

$$
\dot{x}=-c|\dot{u}| x+\Phi^{\prime}(u) \dot{u},
$$

where $c>0, \Phi \in C^{1}(\mathbb{R}, \mathbb{R})$, input $u \in W^{1, \infty}\left(\mathbb{R}_{+}, \mathbb{R}\right)$, and $\Phi^{\prime}(u)=d \Phi(\alpha) /\left.d \alpha\right|_{\alpha=u}$.

It can be easily shown that Condition 1 in Lemma 4.1 is satisfied. Equation (17) can be written as

$$
\begin{aligned}
\dot{x} & =f(x, u) g(\dot{u}) \\
& =\left[-c x+\Phi^{\prime}(u) c x+\Phi^{\prime}(u)\right]\left[\begin{array}{c}
\max \{0, \dot{u}\} \\
\min \{0, \dot{u}\}
\end{array}\right] .
\end{aligned}
$$

Clearly, the function $g$ is of class $\lambda=1$ and satisfies Condition 2 in Lemma 4.1. This implies that the operator which maps $(u, x(0))$ to $x$ is consistent and $\sigma_{\gamma}$ is independent of $\gamma$

Lemma 4.2. Consider the semilinear Duhem model with $m=1$, and $\lambda=1$

$$
\begin{aligned}
\dot{x} & =(A x+B u+C) g(\dot{u}), \\
x(0) & =x_{0},
\end{aligned}
$$

where $A=\left[\begin{array}{llll}a_{1} & a_{2} & \cdots & a_{r}\end{array}\right] \neq \mathbf{0}, B$, and $C$ are $1 \times r$ row vectors, state $x \in \mathbb{R}$, function $g \in C^{0}\left(\mathbb{R}, \mathbb{R}^{r}\right)$ is of class $\lambda=1$, and non-constant input $u \in W^{1, \infty}\left(\mathbb{R}_{+}, \mathbb{R}\right)$. Denote

$$
\begin{aligned}
& \lim _{\kappa \rightarrow 0+} \frac{g(\kappa)}{|\kappa|}=G_{+}^{*}=\left[\begin{array}{llll}
G_{+, 1}^{*} & G_{+, 2}^{*} & \cdots & G_{+, r}^{*}
\end{array}\right], \\
& \lim _{\kappa \rightarrow 0-} \frac{g(\kappa)}{|\kappa|}=G_{-}^{*}=\left[\begin{array}{llll}
G_{-, 1}^{*} & G_{-, 2}^{*} & \cdots & G_{-, r}^{*}
\end{array}\right] .
\end{aligned}
$$

For any $i \in\{1,2, \ldots, r\}$, assume that

$$
\begin{aligned}
& G_{+, i}^{*}, G_{-, i}^{*} \geq 0 \text { whenever } a_{i}<0, \\
& G_{+, i}^{*}, G_{-, i}^{*} \leq 0 \text { whenever } a_{i}>0 .
\end{aligned}
$$

Suppose that there exists some $i_{0} \in\{1,2, \ldots, r\}$ such that

$$
a_{i_{0}} \neq 0 \text { and }\left|G_{+, i_{0}}^{*}\right|+\left|G_{-, i_{0}}^{*}\right|>0 \text {. }
$$

Then
- There exist $E, \gamma_{1}>0$ such that $\left\|x_{\gamma}\right\|_{\infty} \leq E, \forall \gamma>\gamma_{1}$. 3

- There exists a function $q_{u} \in W^{1, \infty}\left(\mathbb{R}_{+}, \mathbb{R}\right)$ such that $\left\|\sigma_{\gamma}-q_{u}\right\|_{1, \infty} \rightarrow 0$, as $\gamma \rightarrow \infty$ where $\|\cdot\|_{1, \infty}$ is the norm of the Banach space $W^{1, \infty}\left(\mathbb{R}_{+}, \mathbb{R}^{m}\right)$.

- $q_{u}(0)=x_{0}$. Furthermore, for almost all $t \geq 0$, we have $\dot{q}_{u}(t)=\left(A q_{u}(t)+B u(t)+C\right) g^{*}(\dot{u}(t))$, where the function $g^{*} \in C^{0}(\mathbb{R}, \mathbb{R})$ is defined as in equation (6), i.e.

$$
g^{*}(\vartheta)= \begin{cases}\vartheta G_{+}^{*} & \vartheta \geq 0 \\ -\vartheta G_{-}^{*} & \vartheta<0\end{cases}
$$

Example. The LuGre model is described by [26]:

$$
\begin{aligned}
& \dot{x}=\dot{u}-\theta \frac{|\dot{u}|}{\mu(\dot{u})} x=\left[\begin{array}{ll}
1 & x
\end{array}\right]\left[\begin{array}{c}
\dot{u} \\
-\theta \frac{|\dot{u}|}{\mu(\dot{u})}
\end{array}\right]=f(x) g(\dot{u}) \\
& x(0)=x_{0}, \\
& F=\theta x+c_{1} \dot{x}+c_{2} \dot{u},
\end{aligned}
$$

where parameters $\theta, c_{1}, c_{2}>0$ are respectively the stiffness, damping, and viscous friction coefficients, $x \in \mathbb{R}$ is the average deflection of the bristles, $x_{0} \in \mathbb{R}$ is the initial state, $u \in W^{1, \infty}\left(\mathbb{R}_{+}, \mathbb{R}\right)$ is the relative displacement and is the input of the system, and $F$ is the friction force and is the output of the system. The function $\mu: \mathbb{R} \rightarrow \mathbb{R}$ is defined as $\mu(\alpha)=F_{C}+\left(F_{S}-F_{C}\right) e^{-\left|\alpha / v_{s}\right|}, \forall \alpha \in \mathbb{R}$, where $F_{C}>0$ is the Coulomb friction force, $F_{S}>0$ is the stiction force, and $v_{s} \in \mathbb{R}$ is the Stribeck velocity.

The LuGre model can be written in the form of the system (18)-(19) with $A=\left[\begin{array}{ll}a_{1} & a_{2}\end{array}\right]=\left[\begin{array}{ll}0 & 1\end{array}\right], B=\left[\begin{array}{ll}0 & 0\end{array}\right]$, and $C=\left[\begin{array}{ll}1 & 0\end{array}\right]$. Furthermore, it can be easily checked that Conditions (20)-(22) are satisfied. Thus, Lemma 4.2 implies that $\left\|\sigma_{\gamma}-q_{u}\right\|_{1, \infty} \rightarrow 0$, as $\gamma \rightarrow \infty$, where the function $q_{u} \in W^{1, \infty}\left(\mathbb{R}_{+}, \mathbb{R}\right)$ are defined for all $t \geq 0$ as

$$
q_{u}(t)=x_{0}+\int_{0}^{t}\left(\dot{u}(\tau)-\frac{\theta|\dot{u}(\tau)|}{F_{S}} q_{u}(\tau)\right) d \tau .
$$

\footnotetext{
$3 x_{\gamma}$ is the output of the system (18)-(19) when we use the input $u \circ s_{\gamma}$ instead of the input $u$ (see system (3)-(4))
} 

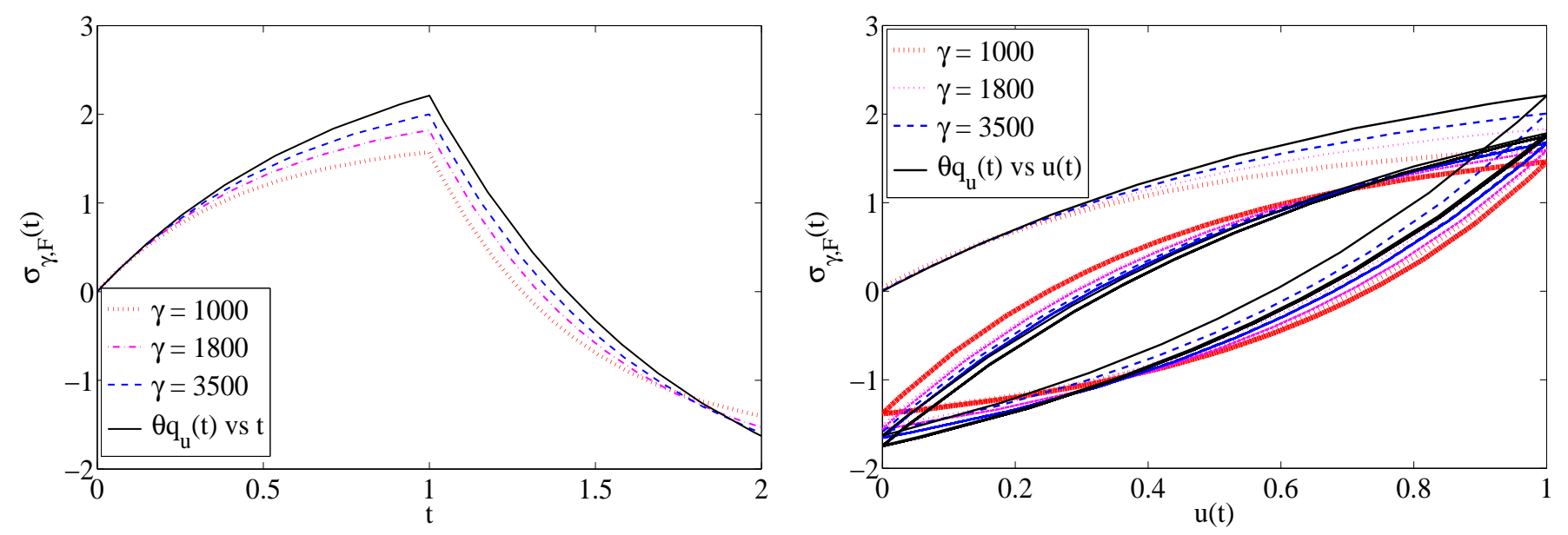

Fig. 2: Left $\sigma_{\gamma, F}(t)$ versus $t$. Right $\sigma_{\gamma, F}(t)$ versus $u(t)$.

Also, there exist some $E, \gamma_{1}>0$ such that for all $\gamma>\gamma_{1}$, the solution of (23)-(24) is global with $\left\|x_{\gamma}\right\|_{\infty} \leq E$.

Now, the following analysis is not a part of Lemma 4.2, but it follows straightforwardly from it. Let $F_{\gamma}$ be the output of the system when we use the input $u \circ s_{\gamma}$ instead of $u$. We obtain from (25) for almost all $t \geq 0$ that

$$
F_{\gamma}(t)=\theta x_{\gamma}(t)+c_{1} \dot{x}_{\gamma}(t)+\frac{c_{2}}{\gamma} \dot{u} \circ s_{\gamma}(t), \forall \gamma>0,
$$

which leads to

$$
\sigma_{\gamma, F}(t)=\theta \sigma_{\gamma}(t)+c_{1} \frac{\dot{\sigma}_{\gamma}(t)}{\gamma}+\frac{c_{2}}{\gamma} \dot{u}(t), \forall \gamma>0
$$

where $\sigma_{\gamma, F}: \mathbb{R}_{+} \rightarrow \mathbb{R}^{m}$ is defined as $\sigma_{\gamma, F}(t)=$ $F_{\gamma}(\gamma t), \forall \gamma>0, \forall t \geq 0$.

Since $\left\|\sigma_{\gamma}-q_{u}\right\|_{1, \infty} \rightarrow 0$, as $\gamma \rightarrow \infty$, we have

$$
\begin{aligned}
& \lim _{\gamma \rightarrow \infty}\left\|\sigma_{\gamma}-q_{u}\right\|_{\infty}=0, \\
& \lim _{\gamma \rightarrow \infty}\left\|\dot{\sigma}_{\gamma}-\dot{q}_{u}\right\|_{\infty}=0 .
\end{aligned}
$$

Thus, we obtain from the boundedness of $\dot{u}$ and equation (27) that $\lim _{\gamma \rightarrow \infty}\left\|\sigma_{\gamma, F}(t)-\theta q_{u}\right\|_{\infty}=0$, which means that the operator which maps input $u$ and initial state $x_{0}$ to output $F$ is consistent.

The conclusion of the analysis is that the hysteresis loop of the LuGre model is $\left\{\left(\theta \dot{q}_{u}(t), u(t)\right), t \geq 0\right\}$, where $q_{u}$ is given in (26). Observe that this conclusion has been obtained due to the convergence of $\sigma_{\gamma}$ in $W^{1, \infty}\left(\mathbb{R}_{+}, \mathbb{R}\right)$.

Simulations: Take $\theta=4 \mathrm{~N} / \mathrm{m}, v_{S}=0.001 \mathrm{~m} / \mathrm{s}, F_{S}=3$ $\mathrm{N}, F_{C}=1 \mathrm{~N}, c_{1}=1 \mathrm{~N}-\mathrm{s} / \mathrm{m}, c_{2}=1 \mathrm{~N}-\mathrm{s} / \mathrm{m}$, and $x(0)=0$ $\mathrm{N}$. Let $u \in W^{1, \infty}\left(\mathbb{R}_{+}, \mathbb{R}\right)$ be a function of period 2 that is measured in meters such that $u(t)=t, \forall t \in[0,1] \mathrm{s}$, and $u(t)=2-t, \forall t \in[1,2] \mathrm{s}$. Figure 2 left shows the uniform convergence of $\sigma_{\gamma, F}$ to $\theta q_{u}$ as $\gamma \rightarrow \infty$. Figure 2 right shows that the graphs $\left\{\left(\sigma_{\gamma}(t), u(t)\right), t \geq 0\right\}$ converge to the hysteresis loop $\left\{\left(\theta q_{u}(t), u(t)\right), t \geq 0\right\}$.

\section{CONCLUSION}

This paper presented a classification of the possible Duhem models in terms of their consistency with the hysteresis behavior. Three classes of models have been considered in relation with the range of a parameter $\lambda$. For $\lambda>1$ it has been shown that the corresponding generalized Duhem model does not represent a hysteresis behavior. For $0<\lambda<1$ it has been shown that the semilinear Duhem model is not compatible with a hysteresis behavior. In all other cases, necessary conditions and sufficient ones have been derived to insure the consistency of the Duhem model with the hysteresis property. Numerical simulations have been carried out to illustrate the obtained results.

\section{ACKNOWLEDGMENT}

Supported by grant DPI2011-25822 of the Spanish Ministry of Economy and Competitiveness.

\section{REFERENCES}

[1] R.A. Adams, and J.J. F. Fournier, Sobolev spaces, Elsevier, 2003.

[2] F.B. Adda, and J. Cresson, "About non-differentiable functions", Journal of Mathematical Analysis and Applications, vol. 263, no. 2, pp. 721-737, 2001.

[3] D.S. Bernstein, "Ivory ghost [ask the experts]", IEEE Control Systems, vol. 27, no. 5, pp. 16-17, 2007.

[4] R. Bouc, "Modèle mathématique d'hystérésis", Acustica, vol. 24, pp. 16-25, 1971.

[5] M. Brokate, and J. Sprekels, Hysteresis and phase transitions, Springer-Verlag, New York, 1996.

[6] P.R. Dahl, "A solid friction model", The Aerospace Corporation El Segundo, TOR-0158 (3107-18), Clifornia, 1968.

[7] P. Duhem, "Die dauernden aenderungen und die thermodynamik", Zeitschrift fur Physikalische Chemie, vol. 22, pp. 543-589, 1897.

[8] A.F. Filippov, Differential equations with discontinuous right-hand sides, Kluwer, (1988).

[9] M.L. Hodgdon, "Applications of a theory of ferromagnetic hysteresis", IEEE Transactions on Magnetic, vol. 24, no. 1, pp. 218- 221, 1988. 
[10] M.L. Hodgdon, "Mathematical theory and calculations of magnetic hysteresis curves", IEEE Transactions on Magnetic, vol. 24, no. 6, pp. 3120-3122, 1988.

[11] F. Ikhouane, "Characterization of hysteresis processes", Mathematics of Control, Signals, and Systems , DOI 0.1007/s00498-012-0099-6.

[12] F. Ikhouane, and J. Rodellar, Systems with hysteresis: analysis, identification and control using the BoucWen model, Wiley, Chichester, UK, 2007.

[13] B. Jayawardhana, R. Ouyang, and V. Andrieu, "Stability of systems with the Duhem hysteresis operator: the dissipativity approach", Automatica, vol. 48, no. 10, pp. 2657-2662, 2012.

[14] H. K. Khalil, Nonlinear systems, Third Edition, Prentice Hall, Upper Saddle River, New Jersey, 2002, ISBN 0130673897.

[15] M. A. Krasnosel'skii, and A. V. Pokrovskii, Systems with hysteresis, Springer-Verlag, Berlin Heidelberg, 1989.

[16] G. Leoni, A First course in sobolev spaces, American Mathematical Society, Providence, RI, 2009.

[17] J.W. Macki, P. Nistri, and P. Zecca, "Mathematical models for hysteresis", SIAM Review, vol. 35, no. 1, pp. 94-123, 1993.

[18] I. Mayergoyz, Mathematical models of hysteresis, Elsevier Series in Electromagnetism, New-York, 2003.

[19] J. Oh, and D.S. Bernstein, "Semilinear Duhem model for rate-independent and rate-dependent hysteresis", IEEE Transactions on Automatic Control, vol. 50, no. 5, pp. 631-645, 2005.

[20] R. Ouyang, V. Andrieu, B. Jayawardhana, "On the characterization of the Duhem hysteresis operator with clockwise input-output dynamics", Systems and Control Letters, vol. 62, no. 3, pp. 286-293, 2013.

[21] A. Padthe, and D.S. Bernstein, "A delay-Duhem model for jump-resonance hysteresis", 46th IEEE Conference on Decision and Control, pp. 1609-1614, 2007.

[22] A.K. Padthe, B. Drincic, J. Oh, D.D. Rizos, S.D. Fassois, and D.S. Berstein, "Duhem modeling of frictioninduced hysteresis", IEEE Control System Magazine, vol. 28, no. 5, pp. 90-107, 2008.

[23] A. Rodriguez, N. Iwata, F. Ikhouane, and J. Rodellar, "Modeling and identification of a large-scale magnetorheological fluid damper", Smart Material and Structures, vol. 18, pp. 374-379, 2009.

[24] A. Visintin, Differential models of hysteresis, Springer-Verlag, Berlin, Heidelberg, 1994.

[25] Y. K. Wen, "Method for random vibration of hysteretic systems", Journal of Engineering Mechanics, vol. 102 , no. 2, pp. 249-263, 1976.

[26] D. Wit, C. Canudas, "A new model for control of systems with friction", IEEE Transactions on Automatic Control, vol. 40, no. 3, pp. 419-425, 1995. 